\title{
Correction: Li, Q.; Liang, S.Y. Incipient Fault Diagnosis of Rolling Bearings Based on Impulse-Step Impact Dictionary and Re-Weighted Minimizing Nonconvex Penalty Lq Regular Technique. Entropy $2017,19,421$
}

\author{
Qing $\mathrm{Li}^{1, *(D)}$ and Steven Y. Liang ${ }^{1,2}$ \\ 1 College of Mechanical Engineering, Donghua University, Shanghai 201620, China \\ 2 George W. Woodruff School of Mechanical Engineering, Georgia Institute of Technology, \\ Atlanta, GA 30332-0405, USA; steven.liang@me.gatech.edu \\ * Correspondence: suesliqing@163.com
}

Received: 5 April 2020; Accepted: 14 April 2020; Published: 23 April 2020

The authors were not aware of some errors and imprecise descriptions made in the proofreading phase, therefore, we wish to make the following corrections to this paper [1]:

On page 3, Section 2, Paragraph 1, the sentence "The first impact approaches a step-like waveform and the second impact resembles an impulse-like waveform," should be read as: "The first impact could be treated as step-like response (i.e., with low frequency components) and the second impact could be treated as impulse-like response (i.e., with high frequency components) [27]."

On page 4, Section 2, Paragraph 5, the sentence " $\tau$ is system damping and $a$ is the peak value ratio of impulse-like to the step-like impact" should be read as: " $\tau$ is system damping and $a$ is the peak value ratio of the impulse-like response to the step-like response [27]".

On page 6, Section 3.2, the Paragraph 3 should be read as: "To overcome the above issue, inspired by the ideas of the unconstrained low-rank matrix recovery in Refs. [35-37] that many successful applications have implemented in the compressed sensing field [29-33], a new re-weighted minimizing nonconvex penalty Lq $(0<q \leq 1)$ regular (R-WMNPLq) method is introduced, which is different from the ones studied in [35-37] where uniform random matrix (URM, i.e., the entries of matrix are random variables with uniform distribution) was used. In this work, the impulse-step impact dictionary is utilized for extracting the fault information from its observation or noisy data. The objective function is as follows:".

On page 7, Table Algorithm 1, the title "Non-Convex-Penalty Smoothed Minimization Lq Algorithm (R-NSMLq)" should be read as "Re-weighted minimizing nonconvex penalty Lq regular (R-WMNPLq)".

On page 7, Equation (12) should be read as $\left(D^{T} D+\operatorname{diag}\left(\frac{q \lambda}{\left(\varepsilon_{k}^{2}+\left\|\alpha^{(k)}[i]\right\|_{2}^{2}\right)^{1-\frac{q}{2}}}\right) \underset{1 \leq i \leq M}{ }\right) \alpha^{(k+1)}=D^{T} b$.

On page 7, Equation (16) should be read as $\left(\varepsilon_{k}^{2}+\|x\|_{2}^{2}\right)^{1-\frac{q}{2}}\left(\varepsilon_{k+1}^{2}+\|y\|_{2}^{2}\right)^{\frac{q}{2}} \leq\left(1-\frac{q}{2}\right)\left(\varepsilon_{k}^{2}+\|x\|_{2}^{2}\right)+$ $\frac{q}{2}\left(\varepsilon_{k+1}^{2}+\|y\|_{2}^{2}\right)$.

On page 7, the sentence "Theorem 1. Error estimation theorem" should be read as: "Theorem 1. Error estimation theorem $[35,36]$ ". The sentence "To prove the theorem 1, the following two lemmas are required" should be read as: "To prove Theorem 1, the following two lemmas (i.e., Lemmas 1 and 2) $[35,36]$ are required". The citation of $[35,36]$ after Lemma 1 (Page 7), Lemma 2 (Page 8) and Proof of Theorem 1 (page 17) should be cited accordingly.

On page 9, Equation (23) should be read as $\frac{q}{2\left(\varepsilon_{k}^{2}+\left|x_{j}^{(k)}\right|^{2}\right)^{1-\frac{q}{2}}} \geq \frac{q}{2\left(\varepsilon_{0}^{2}+\beta^{2}\right)^{1-\frac{q}{2}}}$, and $\frac{1}{C_{4}}=\frac{q}{2\left(\varepsilon_{0}^{2}+\beta^{2}\right)^{1-\frac{q}{2}}}$. 
Due to the addition of new references, the order of the original citations has been changed correspondingly.

The newly added references are as below:

27. Sawalhi, N.; Randall, R.B. Vibration response of spalled rolling element bearing: observations, simulations and signal processing techniques to track the spall size. Mech. Syst. Signal Process. 2011, 25, 846-870.

35. Lai, M.J; Wang, J.Y. An unconstrained $l_{q}$ minimization with $0<q \leq 1$ for sparse solution of underdetermined linear systems. SIAM J. Optim. 2011, 21, 82-101.

36. Lai, M.J.; Xu, Y.Y.; Yin, W.T. Improved iteratively reweighted least squares for unconstrained smoothed $l_{q}$ minimization. SIAM J. Numer. Anal. 2013, 51, 927-957.

37. Wang, Y.; Wang, J.; Xu, Z. On recovery of block-sparse signals via mixed $l_{2} / l_{q}(0<q \leq 1)$ norm minimization. EURASIP J. Adv. Signal Process. 2013, 2013, 76.

These changes do not influence the conclusions of this paper. The authors would like to apologize for any inconvenience caused.

\section{Reference}

1. Li, Q.; Liang, S.Y. Incipient Fault Diagnosis of Rolling Bearings Based on Impulse-Step Impact Dictionary and Re-Weighted Minimizing Nonconvex Penalty Lq Regular Technique. Entropy 2017, 19, 421. [CrossRef]

(C) 2020 by the authors. Licensee MDPI, Basel, Switzerland. This article is an open access article distributed under the terms and conditions of the Creative Commons Attribution (CC BY) license (http://creativecommons.org/licenses/by/4.0/). 